\title{
Transcriptional profiling of fetal hypothalamic TRH neurons
}

\author{
Magdalena Guerra-Crespo ${ }^{1}$, Carlos Pérez-Monter ${ }^{1}$, Sarath Chandra Janga ${ }^{2,6}$, Santiago Castillo-Ramírez ${ }^{3}$, \\ Rosa María Gutiérrez-Rios ${ }^{4}$, Patricia Joseph-Bravo ${ }^{1}$, Leonor Pérez-Martínez ${ }^{5^{*}}$ and Jean-Louis Charli ${ }^{1 *}$
}

\begin{abstract}
Background: During murine hypothalamic development, different neuroendocrine cell phenotypes are generated in overlapping periods; this suggests that cell-type specific developmental programs operate to achieve complete maturation. A balance between programs that include cell proliferation, cell cycle withdrawal as well as epigenetic regulation of gene expression characterizes neurogenesis. Thyrotropin releasing hormone (TRH) is a peptide that regulates energy homeostasis and autonomic responses. To better understand the molecular mechanisms underlying TRH neuron development, we performed a genome wide study of its transcriptome during fetal hypothalamic development.

Results: In primary cultures, TRH cells constitute $2 \%$ of the total fetal hypothalamic cell population. To purify these cells, we took advantage of the fact that the segment spanning -774 to +84 bp of the Trh gene regulatory region confers specific expression of the green fluorescent protein (GFP) in the TRH cells. Transfected TRH cells were purified by fluorescence activated cell sorting, various cell preparations pooled, and their transcriptome compared to that of GFP- hypothalamic cells. TRH cells undergoing the terminal phase of differentiation, expressed genes implicated in protein biosynthesis, intracellular signaling and transcriptional control. Among the transcriptionassociated transcripts, we identified the transcription factors Klf4, Klf10 and Atf3, which were previously uncharacterized within the hypothalamus.

Conclusion: To our knowledge, this is one of the first reports identifying transcripts with a potentially important role during the development of a specific hypothalamic neuronal phenotype. This genome-scale study forms a rational foundation for identifying genes that might participate in the development and function of hypothalamic TRH neurons.
\end{abstract}

\section{Background}

The hypothalamus mediates homeostasis by integrating various endocrine and autonomic responses. Distinct nuclei of the hypothalamus regulate sleep, circadian rhythm, energy homeostasis, sexual behaviors and thermogenesis. Despite extensive studies on the physiological and clinical aspects of hypothalamic function, the molecular mechanisms defining the identity of the neuronal

\footnotetext{
* Correspondence: leonor@ibt.unam.mx; charli@ibt.unam.mx

'Departamento de Genética y Fisiología Molecular, Instituto de

Biotecnología, Universidad Nacional Autónoma de México (UNAM),

Cuernavaca, Morelos, México

${ }^{5}$ Departamento de Medicina Molecular y Bioprocesos, Instituto de Biotecnología, Universidad Nacional Autónoma de México (UNAM),

Cuernavaca, Morelos, México

Full list of author information is available at the end of the article
}

subtypes within each hypothalamic nucleus during development remain poorly defined.

In the murine hypothalamus, five of the neuroendocrine phenotypes [corticotropin-releasing hormone $(\mathrm{CRH})$, thyrotropin releasing hormone (TRH), somatostatin (SS), growth hormone releasing hormone (GHRH), and dopamine (DA) neurons] are generated during partially overlapping periods of time, mainly from the proliferative neuroepithelium of the third ventricle [1]. Cells that produce $\mathrm{CRH}$ are generated between embryonic (E) days 12 and E14, with the peak generation at E13. DA and SS neurons are generated between E11 and E17, while the GHRH and TRH neurons are generated between E11 and E15, with the peak generation at E11 and E13, respectively [2-4].

\section{Biomed Central}


An interesting observation is that subpopulations of neuroendocrine cells coexisting in the same hypothalamic nucleus produce different neuropeptides (e.g. TRH and $\mathrm{CRH})[5,6]$. The distinct neurotransmitter phenotypes do not differ in time of generation and may appear in response to individual differentiation programs involving specific gene networks, as demonstrated for serotoninergic, noradrenergic or dopaminergic phenotypes [7].

The development of the central nervous system (CNS) is achieved through a delicate balance between cell proliferation, subsequent cell cycle withdrawal and differentiation to distinctive neuronal phenotypes. Recent observations have highlighted that both extracellular cues (growth factors, extracellular matrix, Notch-Delta signaling, N-CAMs) and intracellular signals (transcription factors) play pivotal roles in this process [8,9]. In addition, posttranslational histone and/or DNA enzymatic modifications, collectively called epigenetic gene regulation, also govern the process of neurogenesis [10].

In vivo models provide evidence that several transcription factors belonging to the basic helix-loop-helix, homeobox and POU domain families determine the proper establishment and maturation of various neuronal phenotypes within the hypothalamic nuclei [11-13]. In spite of these observations, the inductive signals and final targets of these transcription factors are poorly characterized.

Our group has previously demonstrated that the neurotrophin brain-derived neurotrophic factor (BDNF) increases hypothalamic Trh mRNA expression in rat E17 primary cultures. The BDNF effect is only observed in a population of TRH neurons that express the catalytic isoform of the BDNF receptor, TrkB [14]. In vivo studies have also demonstrated that the expression of the TrkB receptor precedes chronologically that of $\mathrm{TRH}$ in the paraventricular nucleus (PVN) of the rat hypothalamus; the effect of BDNF on Trh mRNA expression can also be observed in primary cultures of PVN neurons [15]. BDNF likely regulates the acquisition and/or maintenance of this phenotype during development. To gain a better understanding of the genes that control differentiation of a specific phenotype in the hypothalamic neurons, we performed a genome-wide study to characterize the transcriptome of hypothalamic TRH neurons during the terminal phase of differentiation. This represented a challenge since the hypothalamic TRH cells constitute only about $2 \%$ of the total cell population. To address this issue, we took advantage of the fact that the -774 / +84 bp regulatory region of the Trh promoter confers tissue specific expression [16] and allows expression of the green fluorescent protein (GFP) in the TRH expressing cells. Purification of the TRH cell population was performed by fluorescence activated cell sorting (FACS) as described previously [17].
In this report, we show that hypothalamic TRH neurons undergoing the terminal phase of differentiation, expressed genes implicated in protein biosynthesis, intracellular signaling, and transcriptional regulation. Among the transcripts enriched in the TRH neurons, we identified three potentially relevant transcription factors: the Krüppel-like factor 4 (Klf4), the transforming growth factor beta [TGFß] inducible early growth response factor (Klf10), also known as Tieg1, and the activating transcription factor 3 (Atf3). To our knowledge, this is the first report identifying these transcription factors during hypothalamic development. Current experiments in our group have shown that Klf4 and Klf10 regulate Trh gene expression. We provide a molecular toolkit via a compendium of expression data that can help unravel mechanisms of hypothalamic TRH neuron development.

\section{Results}

\section{Enrichment of embryonic hypothalamic TRH neurons}

To obtain information about the transcriptome of developing TRH expressing cells, we induced GFP expression in TRH neurons using transfected primary hypothalamic cultures derived from rat embryos of 17 days of gestation. This stage corresponds to the terminal phase of differentiation of the TRH phenotype in the hypothalamus [18]. TRH neurons were enriched by FACS. The transcriptome of the TRH neurons and hypothalamic cells was determined by DNA microarray technology (Figure 1A).

We have previously reported the conditions to efficiently transfect TRH neurons in serum-supplemented cultures; control experiments suggested that most GFP+ cells were TRH neurons [17]. Taking advantage of these conditions, we transfected E17 hypothalamic cultures with a GFP expression vector under the control of the minimal Trh promoter region (phrTRH-GFP) and determined the transfection efficiency by FACS. After $48 \mathrm{~h}$ of transfection, $0.4 \%$ of cells were GFP+ (Figure 1B, middle panel). Preparative cell sorting followed by FACS analysis of the GFP + cell population demonstrated a strong enrichment with approximately $94 \%$ of cells being GFP+ (Figure 1B, lower panel). In general, cell viability was higher than $90 \%$ in all conditions examined as determined by propidium iodide (PI) staining (data not shown).

To corroborate the neuronal identity of the sorted GFP+ cell population, the expression of Trh together with celltype specific markers was examined by RT-PCR assays. GFP + cells were separated from the GFP- cells by FACS $48 \mathrm{~h}$ after transfection. As a control, a mixed cell population consisting of GFP+ and GFP- cells was obtained from sorted transfected cultures without selection $(\mathrm{GFP}+/-)$, whereas non-transfected cells (NT) (at 3 days in vitro) were used to establish the basal levels of mRNA expression. An increase in Trh mRNA levels was observed in the GFP+ cells compared with NT cells; this was also evident 

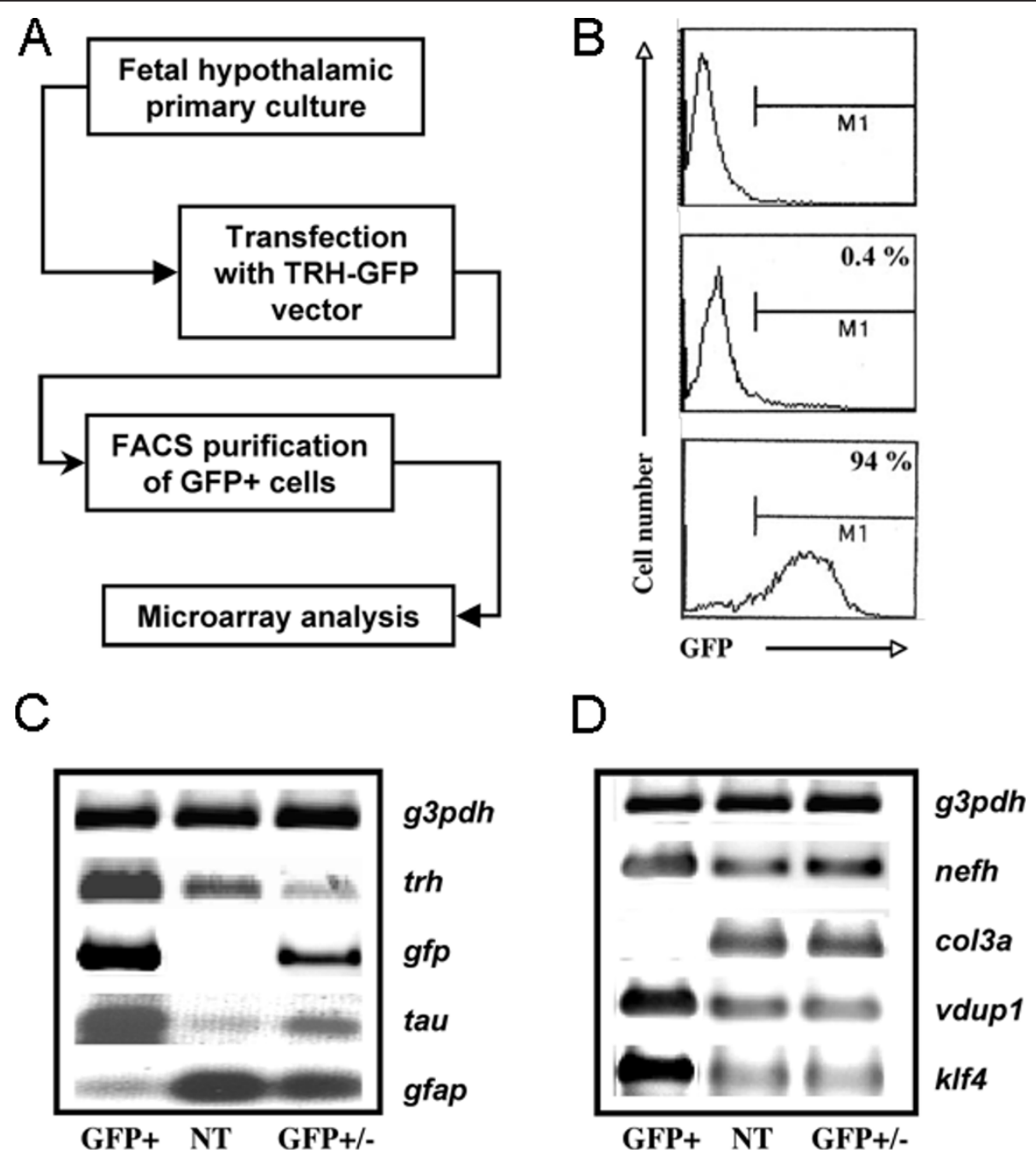

Figure 1 Characterization of the TRH/GFP+ cell population in primary cultures of rat fetal hypothalamic cells. A) Strategy to obtain a neuronal population enriched in TRH cells from primary cultures of fetal (E17) hypothalamic cells. Primary cultures were transfected with a vector driving GFP expression under the control of the Trh promoter (TRH-GFP; referred as phrTRH-GFP in materials and methods) and GFP+ cells were sorted by flow cytometry. GFP+ cells were used to generate the target cRNA to hybridize the U34A array. Non-sorted hypothalamic cells and non-transfected cells were used as control. B) Representative FACS plots indicating the number of GFP+ cells (M1) in a logarithmic scale for $1 \times 10^{4}$ events. The upper panel indicates the distribution of fluorescence in the non-transfected cell population; the middle panel indicates the distribution of fluorescence in cells transfected with phrTRH-GFP; the percentage of GFP+ cells is indicated on the top. Lower panel: after GFP+ cells were isolated by preparative FACS, they were submitted to a second pass through the FACS to determine the percentage of GFP+ cells in the enriched population; it rose to 94\%. C) Several marker transcripts were amplified by RT-PCR. The thyrotropin releasing hormone (trh) and tau transcripts were used as neuronal markers, the glial fibrillary acidic protein ( $g f a p$ ) as glial cell marker and the green fluorescent protein ( $g f p$ ) for transfection control. The glyceraldehyde 3-phosphate dehydrogenase ( $g 3 p d h)$ was used as a control. D) Analysis of transcripts identified in the GFP+ cell population according to microarray data: neurofilament heavy polypeptide (nefh), vitamin D3 up-regulated transcript (vdup 1), and krüppel-like factor 4 (KIf4); the g3pdh was used as internal control. GFP+, transfected purified cells; GFP+/-, transfected non-purified cell population; NT, non-transfected cell population.

with respect to GFP+/- cells (Figure 1C). The increased Trh expression in the isolated GFP+ cells correlated with an increase in the expression of the neuronal marker tau, whereas the expression of the glial cell marker gfap diminished (Figure 1C). As expected, Gfp mRNA was present only in the GFP+ and GFP+/- samples (Figure 1C). These data, together with our previous report [17], indicate that the sorting conditions permitted the enrichment of TRH neurons from a mixed primary cell culture using GFP expression as a marker of Trh proximal promoter activity.

\section{DNA microarray analysis of TRH neurons and} hypothalamic cells

Once we corroborated that TRH cells were enriched in the GFP+ cell population, total RNA from GFP+ cells was isolated from a pool of six independent experiments. 
A pool from three other independent experiments was used to isolate total RNA from GFP+/- and NT cells. These RNA preparations were used to synthesize biotinylated cRNA targets and to screen U34A DNA microarrays.

In the array-screening experiment, two separate aliquots of GFP+ and GFP+/-RNAs, and a single one for NT RNA were used. Target generated from each aliquot was hybridized to an array, generating single (NT) and replicate (GFP+ and GFP+/-) datasets. Within the rat U34A oligonucleotide microarray, we distinguished genes based on whether or not they had a well-characterized gene name in the GenBank database. 7699 probe sets were known genes and 1130 probe sets did not have an assigned gene symbol (expressed sequence tags, ESTs); the total number of transcripts analyzed was 8829 (see http://www.ncbi.nlm. nih.gov/geo/ array).

Analysis of the signal intensity with the Microarray Suite (MAS) 5.0 software (Affymetrix) provides a statistical mean to determine the presence or absence of a gene in a sample. This test is based on the comparison of the hybridization efficiency of the target to its complementary sequence with the cross-hybridization of the target to a mismatch sequence identical to the complementary sequence except for one base. Each gene is represented by 16 probe pairs, with one of the members of each pair containing one mismatch, selected from distinct regions of the gene. The signal values from these probes were used to determine the presence of a gene in the target and a $P$ value calculated from these data. A $P$ value of less than 0.05 was used as a cutoff to consider that a transcript was present and a $P$ value above this threshold indicated that it was absent. Transcripts were considered significantly present or absent based on the following criteria: a) the normalized value from mean differences was higher than the microarray background and, b) the fold change between match and mismatch signals was higher than 2 .

On the basis of this analysis, the percentage of transcripts present in the GFP+ and GFP+/- populations was very similar. In the GFP+ cell population, 41\% (2929 annotated transcripts and 583 ESTs) of the genes represented on the microarray were present, whereas 59\% (4664 annotated transcripts and 398 ESTs) were absent (Additional file 1, Table S1). In the GFP+/- cell population, $41 \%$ of the genes (2941 annotated transcripts and 639 ESTs) were present and 59\% (4756 annotated transcripts and 434 ESTs) were absent. In the NT cell population, 42\% (3014 annotated transcripts and 571 ESTs) of the genes were present and 58\% (4583 annotated transcripts and 410 ESTs) were absent.

\section{Enriched transcripts in the TRH neurons}

To identify the set of enriched transcripts in the GFP+ population, we selected genes with a significant level of differential expression. This involved first grouping the two replicates of the control (i.e. GFP+/-) as well as that of the sorted sample's (i.e. GFP+) expression data. Signal intensities of the two replicates of control and sorted datasets were averaged to represent the expression level of a transcript in the respective control and sorted populations. These averaged intensities were used to calculate the fold enrichment in expression in sorted sample over the control for each transcript. A threshold of more than 2 fold increase or decrease in expression was considered significant to identify transcripts which are enriched in one sample but underrepresented in the other (Table 1).

This analysis revealed that 30 transcripts were enriched in the GFP+ cells (Table 1). As expected, of the 30 transcripts enriched, we identified some transcripts previously associated with a neuronal phenotype, like the neurofilament heavy chain polypeptide (Nefh) [19], a voltage-dependent calcium channel [20], and a nicotinic alpha-receptor subunit [21]. Three of the enriched transcripts corresponded to novel transcripts within the developing hypothalamus, the Krüppel-like 4 transcription factor (Klf4), the TGF $\beta$-inducible early growth response transcription factor (Klf10), also known as Tieg1, and the activator of transcription factor 3 (Atf3). In addition, a transcript up-regulated by vitamin D3 (Vdup1) was enriched in the TRH/GFP+ cell population, suggesting a potential physiological role of this vitamin within the hypothalamic TRH neurons, in agreement with previously reported data [22].

On the other hand, we found that some of the transcripts diminished in TRH/GFP+ cells (i.e. were probably not expressed in TRH cells) were associated with the glial cell phenotype. Among them are the collagen type I and type III (Col3a) [23,24], and the follistatin-like gene, highly expressed in astroglial cells [25]. We also identified transcripts associated with cell cycle regulation, like annexin I, which negatively regulates cyclin D1 gene expression [26] (Additional file 1, Table S2).

We then decreased the microarray threshold to 1.5 fold change to determine if any missing classes of genes can be identified in the different cell populations. We used a heat map presentation and the gene expression profile to establish a hierarchical map based on the similarity of the gene expression values. The first scale, which is associated with a coloured strap, refers to genes with upregulated (red) or down-regulated (green) expression levels in each cell population (Figure 2). The second scale represents the degree of regulation similarity among the genes. A value of zero indicates that the transcripts have the same regulation profile. Figure 2 shows part of the transcripts identified in each cell population. This analysis confirmed the enrichment of various transcripts (Klf4, Atf3, Nefh) in the GFP+ cells. 
Table 1 Specific transcripts enriched in the TRH+/GFP+ cell population in a log2-based analysis $(P$ value $<0.05)$

\begin{tabular}{|c|c|c|c|}
\hline Entrez Gene ID & $\begin{array}{l}\text { Gene Name } \\
\text { Cytoskeleton }\end{array}$ & $\begin{array}{l}\text { Gene } \\
\text { symbol }\end{array}$ & $\log 2$ \\
\hline 24587 & Neurofilament, heavy polypeptide & Nefh & 7.04 \\
\hline 314856 & Transcribed sequence similar to protein Mdm2 & Mdm2 & 3.87 \\
\hline \multicolumn{4}{|c|}{ p53 binding protein homolog } \\
\hline \multicolumn{4}{|c|}{ Cytoplasmic } \\
\hline 288656 & $\begin{array}{l}\text { Transcribed sequence with similarity to ADP- } \\
\text { ribosylation-like factor } 6 \text { interacting protein } 4\end{array}$ & Arl6ip4 & 1.03 \\
\hline \multicolumn{4}{|c|}{ Splicing events } \\
\hline 114121 & Cyclin L & Ccnl1 & 1.45 \\
\hline \multicolumn{4}{|c|}{ Cell signalling } \\
\hline 24373 & Follistatin & Fst & 1.37 \\
\hline 171347 & Methionine adenosyltransferase II, alpha & Mat2a & 1.12 \\
\hline 304268 & RAS-like family 11 member a & Rasl11a & 1.17 \\
\hline 17872 & $\begin{array}{c}\text { Transcribed sequence with similarity to myeloid } \\
\text { differentiation primary response gene } 116\end{array}$ & Myd116 & 1.44 \\
\hline 54305 & Somatostatin receptor subtype 2 & Sstr2 & 2.8 \\
\hline \multicolumn{4}{|c|}{ Transcription } \\
\hline 114505 & Kruppel-like factor 4 (gut) & Klf4 & 2.6 \\
\hline 25389 & Activating transcription factor 3 & Atf3 & 2.05 \\
\hline 81813 & TGFB inducible early growth response & Klf10 & 1.78 \\
\hline 117514 & Upregulated by 1,25-dihydroxyvitamin D-3 & Txnip & 1.32 \\
\hline 314322 & Rat c-fos mRNA & Fos & 2.99 \\
\hline \multicolumn{4}{|c|}{ Cell cycle } \\
\hline 361915 & Hypothetical protein LOC361915 & ND & 1.04 \\
\hline \multicolumn{4}{|c|}{ Metabollic } \\
\hline 154516 & P glucuronosyltransferase 1 family, polypeptide A7C & Ugt1a7c & 1.05 \\
\hline 25617 & Glucose-regulated protein GRP78 & Hspa5 & 1.10 \\
\hline \multicolumn{4}{|c|}{ Neurotransmission } \\
\hline 25302 & Nicotinic receptor alpha 7 subunit & Chrna7 & 1.19 \\
\hline 641625 & Rat genes for vasopressin, oxytocin and a long interspersed repeated & ND & 1.45 \\
\hline ND & Transcribed sequences similar to Cacnb2 and the Nsun 6 gene for NOL1/NOP2/Sun domain family 6 & ND & 1.08 \\
\hline 24766 & Sodium channel, voltage-gated, type II, alpha 1 polypeptide & Scn2a1 & 2.06 \\
\hline \multicolumn{4}{|c|}{ Unknown } \\
\hline Al639457 & Not similitude to any gene found & ND & 2.89 \\
\hline U31866 & Not similitude to any gene found & ND & 1.59 \\
\hline Al639457 & Not similitude to any gene found & ND & 1.59 \\
\hline Al639215 & Not similitude to any gene found & ND & 1.01 \\
\hline M13100 & Not similitude to any gene found & & 1.02 \\
\hline 363278 & Transcribed sequence simmilar to ATG16 autophagy related 16-like 1 (S. cerevisiae) & Atg1611 & 1.08 \\
\hline M13100 & Unknown protein & ND & 1.18 \\
\hline M13101 & Unknown protein & ND & 1.29 \\
\hline M13100 & Not similitude to any gene found & ND & 1.16 \\
\hline
\end{tabular}

ND, non determined

To validate the microarray data, we performed RT-PCR analyses for some of the transcripts presumably enriched in the GFP+ cell population. The levels of mRNAs for Nefh, Vdup1, and Klf4 were increased in the purified population when compared to the NT or GFP+/- cell populations (Figure 1D). On the contrary, the glial phenotype associated transcripts, Gafp and Col3a, were absent in the GFP+ cell population (Figure 1C and 1D). These results confirmed our microarray data.

\section{Transcripts unique to the TRH neurons}

To further breakdown the microarray data, a second method of analysis of the original signal intensities derived from the MAS software analysis was performed 


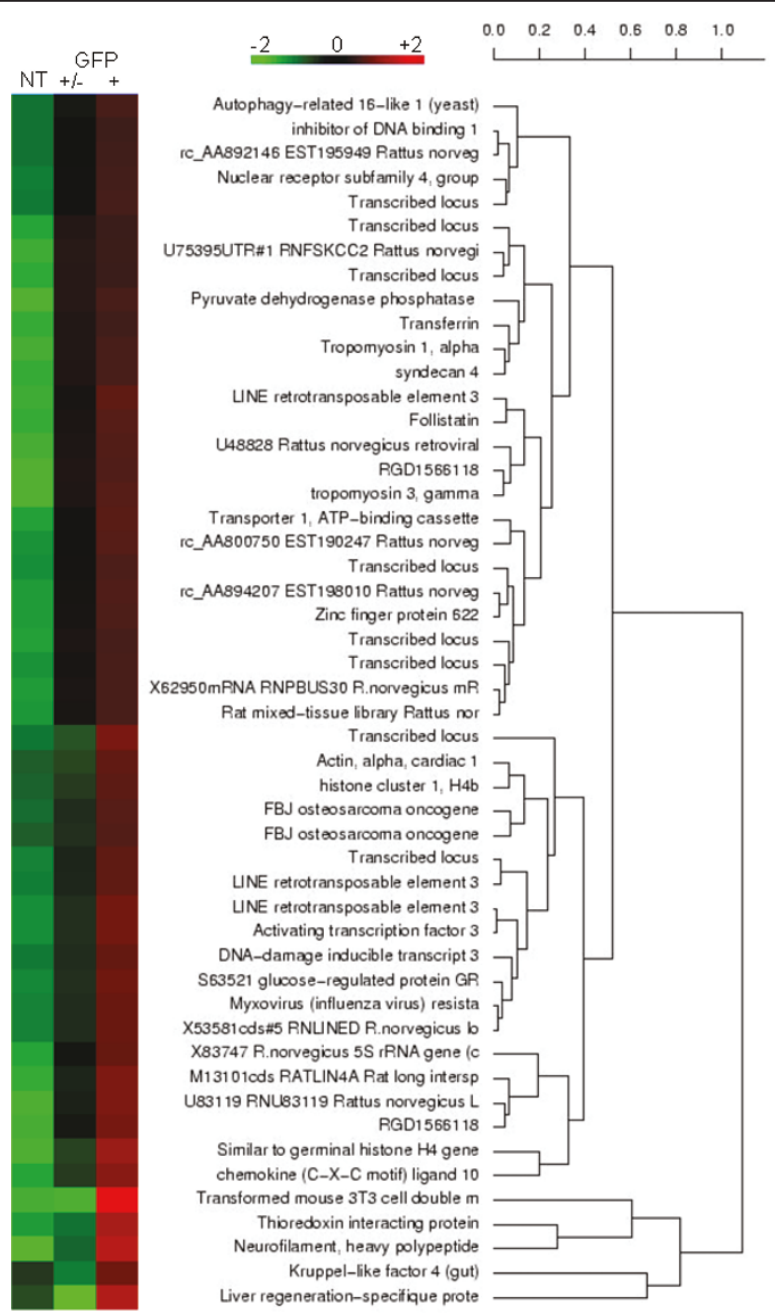

Figure 2 Hierarchical clustering of differently expressed transcripts in hypothalamic cells. The color strap indicates degrees of up- (red) and down- (green) regulation of gene expression. The dendrogram at the right shows the grouping of genes according to their similarity in expression profiles. A value of zero indicates that the genes have the same regulation profile. The transfected purified (GFP+) cell population was compared to the transfected non-purified (GFP+/-) or non-transfected (NT) cell populations in a $\log _{2}$-based threshold. The data correspond to transcripts enriched in the GFP+ cells only. The gene hierarchical cluster was performed using the multiple group analysis and the CLARA algorithm as described [54].

using a stringent $P$-value. This approach allowed us to identify transcripts present in the different cell populations with a high degree of certainty. Using a $P$ value of $<0.001$, we identified a total of 1864 and 1701 transcripts whose presence in the two GFP+/-replicates was significant. Similarly, we identified 1776 and 1714 transcripts in the replicate samples for the GFP+ cell population. In the NT cell population, we identified 1925 transcripts.

In order to identify the transcripts that were present in both replicates and to reduce the false positive rate in the detection of expressed transcripts, we defined the representative set of each sample as that containing transcripts significantly expressed in both replicates according to the $P<0.001$ threshold. This resulted in 1600 [of which 288 were ESTs (Additional file 1, Table S4)] transcripts as representative of the GFP+ cell population (Figure 3 and Additional file 1, Table S3) and 1630 transcripts for the GFP+/- cell population. As shown in Figure 3, the overlap between the three cell populations indicates that 1361 transcripts were common to the three populations, whereas 112 transcripts were common to the GFP+ and GFP+/- cell populations but not expressed in the NT cell population. This comparison also shows that 51 transcripts were unique to the GFP+/- cell population, while 50 transcripts were unique to the GFP+ cell population at these thresholds. It should be noted that in this context unique transcripts refer to those transcripts that are uniquely detected in one or more populations shown in the Venn diagram, as they are likely to be expressed in 


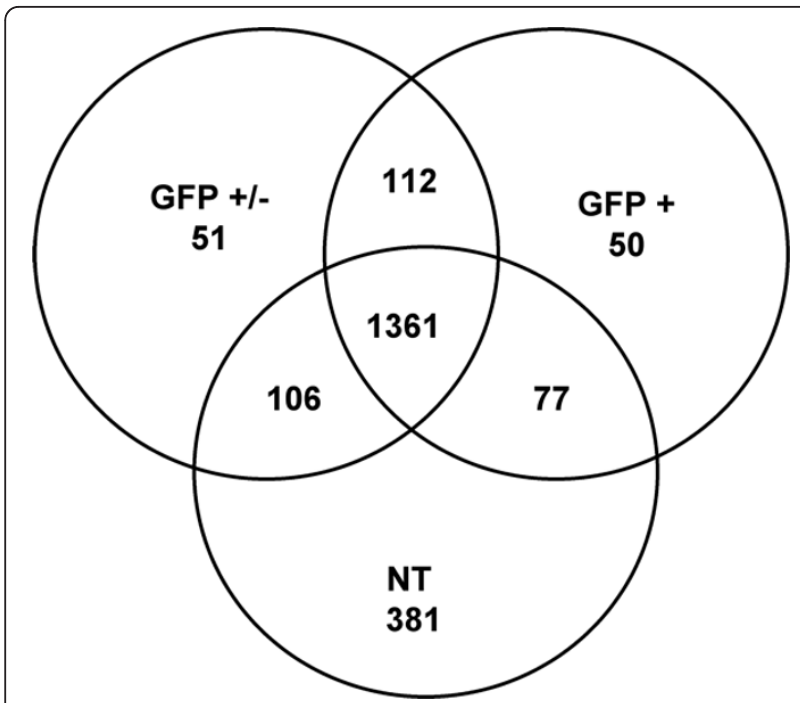

Figure 3 Venn diagram showing the number of transcripts identified present in each cell population. The circles represent a specific cell population with the numbers representing the genes present in each sample $(P<0.001)$. Numbers in the outer portion of each circle are specific to that sample. The number in the innermost portion of the diagram represents transcripts expressed in the three populations (1361). Likewise, the numbers in the portions of the diagram shared by two circles represent genes expressed in those samples but not in the exclusive sample.

undetectable levels at these thresholds in other compared populations. According to their GenBank annotations, several of these 50 transcripts (unique to GFP+ cells) are related to neuronal phenotype, e.g. synaptojanin 1 (Synj1); to translation machinery, e.g. eukaryotic translation initiation factor 3 subunit 9 (Eif3s9), ribosomal protein L27 (Rpl27); to basal metabolic machinery, e.g. acylCoA synthetase long-chain family member 5 (Acsl5), solute carrier family 37 member 4 (Slc37a4); to cell signaling, e.g. the serine-threonine kinase 38 (Stk38); in addition, transcripts encoding proteins with RNA-processing properties were also observed, i.e. the nuclear transcription factor Y gamma (Nfyc), the splicing factor arginine/serine-rich 10 (Sfrs10) and the Y box protein 1 $(Y b x 1)$. Transcripts related to CNS development were also identified, i.e. neurofilament heavy chain polypeptide (Nefh), and the nuclear factor I/B (Nifb). A transcript with chromatin remodeling properties, the transformation/transcription domain-associated protein (Trapp) was also identified (Table 2). These transcripts may play a critical role in the fetal development of hypothalamic TRH neurons.

\section{Discussion}

Events occurring during development are tightly coupled to gene expression regulation. Some specific genes have been related to the establishment of neuronal phenotypes within the hypothalamic area. Particularly, the transcription factors Otp, Sim1, Sim2, as well as Brn2 have important roles in hypothalamic architecture (reviewed in $[27,28])$. The absence of either of these transcription factors during embryonic development leads to anatomical and molecular hypothalamic impairment and consequently, to the complete lack of expression of specific hypothalamic peptides [11-13]. However, the signalling pathways regulating the activity of these transcription factors and their target genes have not been established. To obtain some insight into the molecular mechanisms regulating Trh expression and/or TRH neuron growth during development, we determined elements of the gene expression profile of fetal hypothalamic cells enriched in TRH neurons using the DNA microarray technology. Our approach does not necessarily identify genes relevant for birth or migration, but should capture genes important for late developmental events involving TRH neuron specification and function. Here, we report that FACS enriched TRH neurons, previously cultured for 3 DIV, can be successfully used to characterize elements of their transcriptome. The database generated from this analysis allowed us to identify some transcripts, including several transcription factors, as novel candidates to regulate hypothalamic Trh gene expression or TRH neuron growth during the terminal phase of development. Among the transcripts enriched in the GFP+ cells, we identified three transcription factors whose expression has not been previously reported within the hypothalamus in vivo. These transcripts include the zinc finger domain-containing transcription factor Klf4 [29-32], the TGF $\beta$-inducible early growth response transcription factor (Klf10) [33,34], and the activating transcription factor 3 (Atf3) [35]; these are important regulators of cell differentiation and proliferation in different systems. Recently, these transcription factors have been identified as NGFresponsive immediate early genes during PC12 cell differentiation [36].

Experiments performed in our group have corroborated the relevance of Klf4 for Trh gene expression. Klf4 mRNA is expressed in the embryonic rat hypothalamus, coincident with the establishment of the TRH phenotype; in the neonatal rat hypothalamus, Klf4 is expressed in the PVN, the source of hypophysiotropic TRH. Klf4 binds to the Trh promoter either in vitro or in vivo during fetal hypothalamic development. In addition, Klf4 regulates hypothalamic Trh promoter activity both in vitro and in vivo during development. Accordingly, Trh expression is down-regulated at E15 in the hypothalamus of Klf4 deficient mice, resulting in diminished bioactive peptide level. These data demonstrate that Klf4 is a key molecule within the differentiation program of the hypothalamic TRH phenotype [37]. 
Table 2 Unique transcripts identified in the TRH+/GFP+ cell population using a rigorous threshold of $P<0$

\begin{tabular}{|c|c|c|}
\hline Gene ID & Gene Name & Gene symbol \\
\hline S49760_g_at & 140866 & ND \\
\hline H31982_at & ND & ND \\
\hline AA849648_at & ND & ND \\
\hline X83747 & ND & ND \\
\hline AA800750 & ND & ND \\
\hline AA891631 & ND & ND \\
\hline Al104567 & Actin alpha cardiac 1 & Actc1 \\
\hline AA892318 & ADP-ribosylation factor-like 6 interacting protein 4 & Arl6ip4 \\
\hline AA892821 & $\begin{array}{l}\text { Aldo-keto reductase family } 7 \text {, member A2 } \\
\text { (aflatoxin aldehyde reductase) }\end{array}$ & Akr7a2 \\
\hline Al639209 & Ariadne homolog 2 (Drosophila) (predicted) & Arih2_predicted \\
\hline Al103671 & ATPase, $\mathrm{Ca}++$ transporting, plasma membrane 1 & Atp2b1 \\
\hline M60921 & B-cell translocation gene 2, anti-proliferative & Btg2 \\
\hline AA892271 & BCL2-like 13 (apoptosis facilitator) (predicted) & Bcl2|13_predicted \\
\hline Al639371 & Calponin 3, acidic & Cnn3 \\
\hline U66471 & Cell growth regulator with ring finger domain 1 & Cgrrf1 \\
\hline L23148 & chr1:175179191-175179595 (+) & Y box protein 1 \\
\hline M13101 & C-reactive protein, pentraxin-related & Crp \\
\hline Al180350 & CTD-binding SR-like protein rA9 & LOC245925 \\
\hline$x 62160$ & Dynactin 1 & Dctn1 \\
\hline AA87520 & Eukaryotic translation initiation factor 3, subunit 9 (eta) & Eif3s9 \\
\hline AA858520 & Follistatin & Fst \\
\hline Al639457 & GTP cyclohydrolase 1 & Gch \\
\hline AA799888 & Mitochondrial ribosomal protein $L 40$ & Mrpl40 \\
\hline X62950 & MRNA (pBUS30) with repetitive elements & - \\
\hline AA875126 & Myosin IG & Myolg \\
\hline AA818677 & Neurofilament, heavy polypeptide & Nefh \\
\hline AA859994 & Nuclear factor I/B & Nfib \\
\hline U17254 & Nuclear receptor subfamily 4, group A, member 1 & Nr4a1 \\
\hline AA875121 & Nuclear transcription factor-Y gamma & Nfyc \\
\hline AF040954 & Protein phosphatase 1 , regulatory subunit 10 & Ppp1r10 \\
\hline Al070277 & Proteolipid protein & Plp \\
\hline AA875620 & RGD1566118 (predicted) & - \\
\hline Al176589 & Ribosomal protein L27 & LOC680960 Rpl27 \\
\hline M31018 & RT1 class Ib, locus Aw2 & RT1-Aw2 \\
\hline M10094 & RT1 class Ib, locus Aw2 & RT1-Aw2 \\
\hline Al639167 & Sequestosome 1 & Sqstm1 \\
\hline X05472 & Serine incorporator $1 / / /$ hypothetical protein LOC498354 & $\begin{array}{l}\text { LOC498354 } \\
\text { Serinc1 }\end{array}$ \\
\hline AA800712 & Serine/threonine kinase 38 & Stk38 \\
\hline AF080468 & Solute carrier family 37 (glycerol-6-phosphate transporter), member 4 & Slc37a4 \\
\hline Al639294 & Sparc/osteonectin, cwcv and kazal-like domains proteoglycan 2 (predicted) & Spock2_predicted \\
\hline AA955859 & Splicing factor, arginine/serine-rich 10 (transformer 2 homolog, Drosophila) & Sfrs10 \\
\hline U45479 & Synaptojanin 1 & Synj1 \\
\hline Al014169 & Thioredoxin interacting protein & Txnip \\
\hline AA893320 & $\begin{array}{c}\text { Transcribed locus (Mus musculus ubiquitin specific peptidase } 7 \text { (Usp7). Mus musculus } 10 \text { days neonate cerebellum } \\
\text { CDNA }\end{array}$ & Usp7 \\
\hline AA800693 & $\begin{array}{c}\text { Transcribed locus, strongly similar to XP_579966.1 hypothetical protein XP_579966 [Rattus norvegicus] (Mus } \\
\text { musculus pyridoxal (pyridoxine, vitamin B6) kinase) }\end{array}$ & - \\
\hline AA800513 & Transformation/transcription domain-associated protein (predicted) & Trrap_predicted \\
\hline Al639488 & Transformed mouse 3 T3 cell double minute 2 homolog (mouse) (predicted) & Mdm2_predicted \\
\hline M92074 & Troponin I type 3 (cardiac) & Tnni3 \\
\hline Al639320 & V-raf murine sarcoma 3611 viral oncogene homolog & Araf \\
\hline AA894321 & WD repeat and FYVE domain containing 1 & Wdfy1 \\
\hline
\end{tabular}


TRH/GFP+ cells are enriched with another member of the Krüppel-like family of transcription factors, Klf10. Experimental evidences show that Klf10 is a positive regulator of Trh promoter activity in primary cultures of fetal hypothalamic cells and that its expression coincides with the establishment of the hypothalamic TRH neurons in vivo [38]. Klf10 binds to the Trh promoter in vivo during hypothalamus development (MartínezArmenta M. et al. unpublished).

Previous reports have demonstrated that Klf4, Klf10 and Atf3 expression is regulated by TGF $\beta$ [39-41]. Since TGF $\beta$ isoforms and their receptors are expressed in the hypothalamus [42], we have analyzed the effect of this cytokine in primary hypothalamic cultures. TGF $\beta-2$ increased Trh mRNA expression by regulating Trh promoter activity [38]. Furthermore, TGF $\beta-2$ induced Klf10 mRNA in primary hypothalamic cultures similarly to Trh mRNA. Finally, Klf10 was bound to the rat Trh promoter in response to TGF $\beta$ in vitro (Martínez-Armenta M. et al. unpublished). Therefore, another of the transcription factors identified in the microarray screening appears critical for Trh expression.

In this study, we used independent preparations of GFP + cells that were pooled to identify transcripts enriched in TRH neurons. Using independent preparations of GFP + cells not only decreases the false positives that are possibly identified if only one preparation is used but also enriches the relative concentration of the TRH neuron specific transcripts compared to the background sample. Experimental designs using pooling samples are often performed to reduce the effects of biological variation, making substantive differences easier to find. Consequently, pooling samples has been used in many genome-wide-based studies to provide an accurate and reliable estimate of specific gene expression patterns, DNA methylation profiling, identifying biomarkers, etc. in a specific cell population [43]. Whether all the transcripts identified in this study are truly enriched in TRH hypothalamic neurons in vivo, and whether they are expressed into functional proteins, is not yet known, except for the evidence mentioned above for two transcripts. Under our experimental conditions, TRH neurons passed through a stage of differentiation in vitro; thus, the procedure used in the present study might have altered some aspects of the expression profile of the neurons, leading to some false positives. However, the results obtained with Klf4 and Klf10 (see previous paragraphs) suggest that our data should be a useful guide to study TRH neuron differentiation. However, since TRH neurons are localized in various hypothalamic nuclei [44], each identified transcript may be either enriched in a specific TRH neuron type, or in multiple types.

Previous studies have indicated that each neuronal phenotype possesses a specific differentiation program, in which in addition to transcription factors [7], epigenetic modifications and non-coding RNA expression play pivotal roles. The analysis of the transcriptome of hypothalamic TRH neurons allowed us to derive experimental data that indicate that Klf4 and Klf10 are important regulators of Trh gene expression during the hypothalamus development. Co-activators, such as the histone acetyltransferases (p300 and CBP), or co-repressors, such as histone deacetylases (mSin3a), can regulate Klf4 and Klf10 transcriptional activity [45]. Therefore, we propose that during hypothalamic development Trh gene expression is regulated by extracellular signals (i.e. TGF $\beta$, BDNF) that modulate the accessibility of specific transcription factors (e.g. Klf4 and Klf10) to Trh gene promoter by local histone modifications. To gain further insight into the molecular mechanism regulating hypothalamic neuronal phenotype differentiation, it will be critical to determine the impact of specific epigenetic modifications during hypothalamus development.

\section{Conclusions}

Although the functional importance of the hypothalamus has been demonstrated throughout vertebrates, the molecular mechanisms controlling neurogenesis in this forebrain structure are poorly understood. The hypothalamic TRH peptide has multiple hormonal and autonomous functions. Previous studies have evidenced that pituitary response to TRH is blunted in a number of psychiatric conditions, including schizophrenia, bipolar disorders, alcoholism and depression [46]. Whether specific abnormalities during the differentiation of hypothalamic TRH neurons are associated with such disorders remains unknown. Therefore, knowledge of transcriptional regulation during the course of TRH neuron differentiation might contribute to a better understanding of the molecular mechanisms underlying TRH mediated homeostasis in the adult organism. For this purpose, we performed a genome wide study of hypothalamic TRH neurons during late fetal development. We report novel transcripts within the hypothalamus that may be part of the differentiation program of the TRH neuronal phenotype. These included the transcription factors Klf4, Klf10 and Atf3. Although the role of transcription factors during neuronal differentiation is well accepted, we are only at the brink of understanding how epigenetic mechanisms influence transcriptional activity and the accessibility of transcription factors to bind to ciselements. The identification of transcripts enriched in fetal hypothalamic TRH neurons will guide further studies on the differentiation of this phenotype.

\section{Methods}

\section{Animals}

Wistar rats raised at our animal facility, maintained in standard environmental conditions (lights on between 
0700-1900 h, temperature $21 \pm 2^{\circ} \mathrm{C}$ ) with rat chow and tap water ad libitum. Animal care and protocols followed the guidelines for the use of animals in neuroscience research of the Society for Neuroscience, USA, and were approved by the Animal Care and Ethics Committee of the Instituto de Biotecnología, UNAM.

\section{Cell culture and transfection}

Hypothalamic primary cultures were prepared from E17 rat embryos as previously described [17]. Briefly, pregnant Wistar rats were anesthetized with pentobarbital $(33 \mathrm{mg} /$ kg b.w.) and the embryos removed individually. The hypothalamus was then excised through an imaginary line between both eye and ear superior edge; the dissected area was limited by the optic chiasm and lateral sulcus including mammillary bodies to a 2-3 $\mathrm{mm}$ depth avoiding thalamic area. Hypothalami were dissociated with trypsin (Sigma) and viability monitored by trypan blue exclusion $(95 \%)$. Cells $\left(5 \times 10^{6}\right)$ were plated onto poly-D-lysine (Sigma; $10 \mu \mathrm{g} / \mathrm{ml}$ ) pre-coated $60 \mathrm{~mm}$ Petri dishes in DMEM supplemented with $10 \%$ fetal bovine serum (FBS, GIBCO), 0.25\% glucose (Sigma), $2 \mathrm{mM}$ glutamine (Sigma), $3.3 \mathrm{mg} / \mathrm{ml}$ insulin (Sigma), 1\% antibiotic-antimycotic (GIBCO) and 1\% vitamin solution (GIBCO). Cultures were maintained in a REVCO incubator at $37^{\circ} \mathrm{C}$ in humidified air/7\% $\mathrm{CO}_{2}$.

Twenty-four hours after seeding, cells were transfected essentially as described [17]. In general, $8 \mathrm{mg}$ of branched polyethylenimine (PEI) (600-1000 KDa; Fluka) solution was diluted in $10 \mathrm{ml}$ of water, $\mathrm{pH}$ adjusted to 6.9 with 0.2 $\mathrm{N} \mathrm{HCl}$ and the solution filtered (Millipore, $0.22 \mu \mathrm{m}$ ). PEI $(30 \mu \mathrm{l})$ and plasmid DNA (10 $\mu \mathrm{g})$ were separately diluted to adjust $\mathrm{NaCl}$ to $150 \mathrm{mM}$ in a final volume of $50 \mu \mathrm{l}$, vortexed and incubated for $10 \mathrm{~min}$ at room temperature; subsequently, the polymer solution was added to the DNA, vortexed-mixed, incubated for $10 \mathrm{~min}$ at room temperature followed by the addition of $900 \mu \mathrm{l}$ of serumfree DMEM. The supplemented DMEM was removed from the culture dishes and the transfection mixture was added. After 3 hours incubation, transfection mix was removed and fresh supplemented DMEM was added. Forty-eight hours after transfection, cells were trypsinized (0.25\% trypsin-EDTA) and subjected to FACS.

\section{Plasmid construct}

The minimal Trh promoter $(-776 /+84 \mathrm{bp})$ conferring tissue-specific expression [16] was excised with EcoR1 and BamH1/EcoRV digestion from the pNASS-rTRHLuc expression vector. The Trh promoter fragment sticky ends were filled with Klenow DNA polymerase and subsequently sub-cloned into the SacI/BamH1 sites present on the pACT2 vector. Finally, the Trh promoter fragment was cloned into the SacI/BamHI sites in the phrGFP promoterless expression vector (Stratagene) and sequenced using primers corresponding to the Trh promoter 3' region (5'-ATG CAT AGA TCT TCT AGA TA-3') and to the phrGFP 5'region (3'-TGC AGG CCG GTG TTC TTC AGG A-5'); the resulting plasmid was named phrTRH-GFP.

\section{Fluorescence-activated cell sorting}

For preparative cell sorting, $5 \times 10^{6}$ hypothalamic cells plated on $60-\mathrm{mm}$ dishes were transfected as described above. After 48 h, cells were trypsinized, washed, resuspended in PBS/1\% FBS $\left(10^{7}\right.$ cells $\left./ \mathrm{ml}\right)$ and filtered through a $40 \mu \mathrm{m}$ nylon mesh (BD Bioscience, San Jose, USA). Cells were purified from a pool of five $60-\mathrm{mm}$ dishes using the FACS Vantage (Becton Dickinson, San Jose, $\mathrm{CA})$ and the exclusion method at high speed $(60 \mu \mathrm{l} / \mathrm{min})$. Cells were sorted using the settings previously described [17] and analyzed by analytical flow cytometry as described below. In general, 20,000 GFP+ cells were purified from $5 \times 10^{6}$ cells.

The percentage of GFP + cells before and after purification by preparative cell sorting was determined by analytical flow cytometry using the FACS Vantage. All data acquisition and analyses were performed using the Cell Quest software (Becton Dickinson). To estimate the number of GFP+ cells, a FL1 histogram was generated (FL1, 530/30 nm short pass filter, detects green signal) and positive cells were defined as those cells in the region M1. The percentage of cells in M1 from the empty vector (mock)-transfected cells was subtracted from the percentage of plasmid-transfected cells in M1. Cell viability was determined using propidium iodide (PI) (Sigma) as reported [47]. PI fluorescence was detected with the FL2 emission channel (585/42 $\mathrm{nm}$ band pass filter). The percentage of dead cells was determined as the percentage of PI+ cells in a FL1 versus FL2 plot after subtracting the percentage of PI+ cells from the mock-transfected cells.

\section{DNA microarray analysis}

The microarray analysis was performed as described in the Affymetrix expression analysis technical manual http://www.affymetrix.com. Total RNA (10 $\mu \mathrm{g})$ was extracted from three different cell populations: i) sorted TRH-GFP+ cells (GFP+); ii) TRH-GFP+ and GFP- mixed cells $(\mathrm{GFP}+/-)$ passed through the FACS but not sorted, and iii) non-transfected cells (NT). To obtain a sufficient amount of RNA for each cell population, the number of independent experiments pooled for the GFP+ sample was higher than for the other samples. Therefore, a pool of six independent experiments was used to prepare total RNA from the GFP+ and three independent experiments for GFP+/- or NT cells.

The microarray target was synthesized from total RNA. RNA was reverse-transcribed into double-stranded cDNA with a T7 promoter-containing primer using 
SuperScript II reverse transcriptase, RNase H, and DNA polymerase (Invitrogen). After precipitation with $5 \mathrm{M}$ $\mathrm{NH}_{4} \mathrm{OAc}$ and ethanol, the cDNA was used as a template in a biotin-labeled in vitro transcription reaction (Enzo BioArray, Affymetrix). Resulting target cRNA was collected on RNAeasy columns (QIAGEN, Valencia, CA) and then fragmented for hybridization on the microarrays.

The rat U34A microarray from Affymetrix was used. It contains probes for approximately 7699 well-annotated genes and around 1130 expressed sequence tags (ESTs) from Rattus norvegicus. Probes consist of 16 pairs of 25mer oligonucleotides for each gene. One member of each pair contains a single base point mutation, and the signals of the pairs are compared to assess specificity of hybridization. Biotinylated target cRNA (15 $\mu \mathrm{g})$ was hybridized to the array and then processed using the Affymetrix GeneChip Fluidics Workstation 400. After binding with phycoerythrin-coupled avidin, microarrays were scanned on a Hewlett-Packard Gene Array Scanner (Hewlett-Packard Co., Palo Alto, CA). Data were deposited in the NCBI Gene Expression Omnibus repository http://www.ncbi.nlm.nih.gov/geo/ with the accession number GSE28441.

Results were analyzed using Affymetrix MAS 5.0 software. Individual microarrays were scaled to produce a mean signal intensity of 125 . Iterative comparisons of different microarray datasets were done with MAS 5.0 comparison analysis as previously described with modifications [48]. To determine the expression difference between the $\mathrm{GFP}+$ and GFP+/- cell populations, an additional approach was adopted. This involved grouping the two replicates of the control (i.e, GFP+/-) and the sorted sample (i.e, GFP+). Briefly, signal intensities of the two replicates of control and sorted datasets were averaged to represent the expression level of a transcript in the respective control and sorted populations. These averaged intensities were then used to calculate the fold enrichment in expression in sorted sample over control for each transcript. To identify transcripts that are enriched in one sample but underrepresented in the other, a threshold of more than 2 fold increase or decrease in expression was considered significant.

\section{RT-PCR}

RNA was extracted as previously described [18]. Reverse transcription (RT) was performed with $1 \mu \mathrm{g}$ of RNA using the M-MLV reverse transcriptase (Invitrogen) in the presence of oligo- $\mathrm{dT}_{15}$ primer. PCR was carried out in a total reaction volume of $50 \mu \mathrm{l}$. Primers (Additional file 1, Table S5) were designed using the PRIMER 3 software [49]. In general, PCRs were performed using 25 pmol of each of the specific forward and reverse primers, $1 \mu \mathrm{l}$ of dNTP mix (20 mM each; Boehringer) and $1 / 5$ of the $\mathrm{RT}$ reaction product.

Transcripts amplified by PCR included: Krüppel-like factor 4, collagen type III alpha 1 (Gene ID 84032), upregulated by 1,25 dihydroxyvitamin D-3 (Gene ID 117514), neurofilament heavy chain [50], green fluorescent protein [51], Trh, glyceraldehyde 3-phosphate dehydrogenase (g3pdh) [18], Tau [52] and the glial fibrillary acidic protein [53]. Amplification was performed for 30 cycles except for g3pdh (24 cycles). PCR cycling conditions consisted of one cycle of melt temperature of $94^{\circ} \mathrm{C}$ for $1 \mathrm{~min}$, a primer annealing step at $60^{\circ} \mathrm{C}$ or $64^{\circ} \mathrm{C}$ (Klf4) for $1 \mathrm{~min}$, a polymerization step at $72^{\circ} \mathrm{C}$ for 1 min and a final extension at $72^{\circ} \mathrm{C}$ for $10 \mathrm{~min}$. PCR products were electrophoresed in $2 \%$ agarose gel and bands stained with ethidium bromide.

\section{Acknowledgements}

We thank Affymetrix for the microarray processing and data analysis. We also thank Dr. Jeffrey Bond from the Bioinformatics Core facility at the University of Vermont for the bioinformatics analysis. We are grateful to Dr. Gustavo Pedraza-Alva for insightful scientific discussions, to M. Cisneros, M. Villa and E. Martel for technical support. We also thank S. González for maintaining our rat colony. This work was supported by grants from DGAPA-UNAM (IN227506-3, IN224909) and CONACYT (61208, 42605).

\section{Author details}

${ }^{1}$ Departamento de Genética y Fisiología Molecular, Instituto de Biotecnología, Universidad Nacional Autónoma de México (UNAM), Cuernavaca, Morelos, México. ${ }^{2}$ Laboratory of Molecular Biology, Medical Research Council, Hills Road, Cambridge CB2 0QH, UK. ${ }^{3}$ Centro de Ciencias Genómicas, Universidad Nacional Autónoma de México (UNAM), Cuernavaca, Morelos, México. ${ }^{4}$ Departamento de Microbiología Molecular, Instituto de Biotecnología, Universidad Nacional Autónoma de México (UNAM), Cuernavaca, Morelos, México. ${ }^{5}$ Departamento de Medicina Molecular y Bioprocesos, Instituto de Biotecnología, Universidad Nacional Autónoma de México (UNAM), Cuernavaca, Morelos, México. ${ }^{6}$ Institute for Genomic Biology, University of Illinois at Urbana-Champaign, Urbana, 61801, USA.

\section{Authors' contributions}

MG-C carried out primary cultures and transfection experiments, FACS, prepared the RNA for pooling and validation by RT-PCR, CP-M assisted with the data analysis and participated in writing the manuscript, SCJ performed the majority of the data analysis, SC-R assisted with the data analysis, RMG-R assisted with some data analysis and PJ-B participated in the design of the study, LP-M and J-LC conceived the study, participated in the design and coordination of the study and wrote the manuscript. All authors read and approved the final manuscript.

Received: 13 January 2010 Accepted: 10 May 2011

Published: 10 May 2011

\section{References}

1. Markakis EA: Development of the neuroendocrine hypothalamus. Front Neuroendocrinol 2002, 23:257-291

2. Markakis EA, Swanson LW: Spatiotemporal patterns of secretomotor neuron generation in the parvicellular neuroendocrine system. Brain Res Brain Res Rev 1997, 24:255-291.

3. Angevine JB Jr: Time of neuron origin in the diencephalon of the mouse An autoradiographic study. J Comp Neurol 1970, 139:129-187.

4. Karim MA, Sloper JC: Histogenesis of the supraoptic and paraventricular neurosecretory cells of the mouse hypothalamus. J Anat 1980, 130:341-347. 
5. Sawchenko PE, Imaki T, Vale W: Co-localization of neuroactive substances in the endocrine hypothalamus. Ciba Found Symp 1992, 168:16-30, discussion 30-42

6. Ludwig M, Leng G: Dendritic peptide release and peptide-dependent behaviours. Nat Rev Neurosci 2006, 7:126-136.

7. Goridis $\mathrm{C}$, Rohrer $\mathrm{H}$ : Specification of catecholaminergic and serotonergic neurons. Nat Rev Neurosci 2002, 3:531-541.

8. Edlund T, Jessell TM: Progression from extrinsic to intrinsic signaling in cell fate specification: a view from the nervous system. Cell 1999, 96:211-224.

9. Perez-Martinez $L$, Jaworski DM: Tissue inhibitor of metalloproteinase-2 promotes neuronal differentiation by acting as an anti-mitogenic signal. $J$ Neurosci 2005, 25:4917-4929.

10. Hsieh J, Gage FH: Chromatin remodeling in neural development and plasticity. Curr Opin Cell Biol 2005, 17:664-671.

11. Schonemann MD, Ryan AK, McEvilly RJ, O'Connell SM, Arias CA, Kalla KA Li P, Sawchenko PE, Rosenfeld MG: Development and survival of the endocrine hypothalamus and posterior pituitary gland requires the neuronal POU domain factor Brn-2. Genes Dev 1995, 9:3122-3135.

12. Hosoya T, Oda Y, Takahashi S, Morita M, Kawauchi S, Ema M, Yamamoto M, Fujii-Kuriyama Y: Defective development of secretory neurones in the hypothalamus of Arnt2-knockout mice. Genes Cells 2001, 6:361-374.

13. Michaud JL, DeRossi C, May NR, Holdener BC, Fan CM: ARNT2 acts as the dimerization partner of SIM1 for the development of the hypothalamus. Mech Dev 2000, 90:253-261.

14. Guerra-Crespo M, Ubieta R, Joseph-Bravo P, Charli JL, Perez-Martinez L: BDNF increases the early expression of TRH mRNA in fetal TrkB+ hypothalamic neurons in primary culture. Eur J Neurosci 2001, 14:483-494.

15. Ubieta R, Uribe RM, Gonzalez JA, Garcia-Vazquez A, Perez-Monter C, PerezMartinez L, Joseph-Bravo P, Charli JL: BDNF up-regulates pre-pro-TRH mRNA expression in the fetal/neonatal paraventricular nucleus of the hypothalamus. Properties of the transduction pathway. Brain Res 2007, 1174:28-38.

16. Balkan W, Tavianini MA, Gkonos PJ, Roos BA: Expression of rat thyrotropin releasing hormone (TRH) gene in TRH-producing tissues of transgenic mice requires sequences located in exon 1. Endocrinology 1998 139:252-259.

17. Guerra-Crespo M, Charli JL, Rosales-Garcia VH, Pedraza-Alva G, PerezMartinez $L$ : Polyethylenimine improves the transfection efficiency of primary cultures of post-mitotic rat fetal hypothalamic neurons. $J$ Neurosci Methods 2003, 127:179-192.

18. Perez-Martinez L, Charli JL, Joseph-Bravo P: Development of pro-TRH gene expression in primary cultures of fetal hypothalamic cells. Brain Res Dev Brain Res 2001, 130:73-81.

19. Thyagarajan A, Strong MJ, Szaro BG: Post-transcriptional control of neurofilaments in development and disease. Exp Cell Res 2007, 313:2088-2097.

20. Ross WN, Nakamura T, Watanabe S, Larkum M, Lasser-Ross N: Synaptically activated ca2+ release from internal stores in CNS neurons. Cell Mol Neurobiol 2005, 25:283-295

21. Albuquerque EX, Pereira EF, Alkondon M, Rogers SW: Mammalian nicotinic acetylcholine receptors: from structure to function. Physiol Rev 2009, 89:73-120.

22. Tornquist K, Lamberg-Allardt C: Systemic effects of 1,25-dihydroxyvitamin D3 on the pituitary-hypothalamic axis in rats. Acta Endocrinol (Copenh) 1987, 115:225-228

23. Bunge MB, Williams AK, Wood PM, Uitto J, Jeffrey JJ: Comparison of nerve cell and nerve cell plus Schwann cell cultures, with particular emphasis on basal lamina and collagen formation. J Cell Biol 1980, 84:184-202.

24. Greenberg JH, Foidart JM, Greene RM: Collagen synthesis in cultures of differentiating neural crest cells. Cell Differ 1980, 9:153-163.

25. Zhang G, Ohsawa Y, Kametaka S, Shibata M, Waguri S, Uchiyama Y: Regulation of FLRG expression in rat primary astroglial cells and injured brain tissue by transforming growth factor-beta 1 (TGF-beta 1). J Neurosci Res 2003, 72:33-45.

26. Alldridge LC, Bryant CE: Annexin 1 regulates cell proliferation by disruption of cell morphology and inhibition of cyclin D1 expression through sustained activation of the ERK1/2 MAPK signal. Exp Cell Res 2003, 290:93-107.

27. Michaud $\mathrm{J}$ : The developmental program of the hypothalamus and its disorders. Clin Genet 2001, 60:255-263.
28. Burbach JP, Luckman SM, Murphy D, Gainer H: Gene regulation in the magnocellular hypothalamo-neurohypophysial system. Physiol Rev 2001, 81:1197-1267

29. Shields JM, Christy RJ, Yang WW: Identification and characterization of a gene encoding a gut-enriched Kruppel-like factor expressed during growth arrest. J Biol Chem 1996, 271:20009-20017.

30. Garrett-Sinha LA, Eberspaecher H, Seldin MF, de Crombrugghe B: A gene for a novel zinc-finger protein expressed in differentiated epithelial cells and transiently in certain mesenchymal cells. J Biol Chem 1996, 271:31384-31390.

31. Panigada M, Porcellini S, Sutti F, Doneda L, Pozzoli O, Consalez GG, Guttinger M, Grassi F: GKLF in thymus epithelium as a developmentally regulated element of thymocyte-stroma cross-talk. Mech Dev 1999, 81:103-113.

32. Segre J: Complex redundancy to build a simple epidermal permeability barrier. Curr Opin Cell Biol 2003, 15:776-782.

33. Bieker JJ: Kruppel-like factors: three fingers in many pies. J Biol Chem 2001, 276:34355-34358

34. Cook T, Gebelein B, Belal M, Mesa K, Urrutia R: Three conserved transcriptional repressor domains are a defining feature of the TIEG subfamily of Sp1-like zinc finger proteins. J Biol Chem 1999, 274:29500-29504.

35. Xie J, Roberson MS: 3', 5'-cyclic adenosine 5'-monophosphate response element-dependent transcriptional regulation of the secretogranin II gene promoter depends on gonadotropin-releasing hormone-induced mitogen-activated protein kinase activation and the transactivator activating transcription factor 3. Endocrinology 2008, 149:783-792.

36. Dijkmans TF, van Hooijdonk LW, Schouten TG, Kamphorst JT, Fitzsimons CP, Vreugdenhil E: Identification of new Nerve Growth Factor-responsive immediate-early genes. Brain Res 2009, 1249:19-33.

37. Perez-Monter C, Martinez-Armenta M, Miquelajauregui A, Furlan-Magaril M Varela-Echavarria A, Recillas-Targa F, May V, Charli JL, Perez-Martinez L: The Kruppel-like factor 4 controls biosynthesis of thyrotropin releasing hormone during hypothalamus development. Mol Cell Endocrinol 333:127-133.

38. Martinez-Armenta M, Charli J-L, Perez-Martinez L: The role of Klf10 during differentiation of hypothalamic TRH neurons. 39th annual meeting of the Society for Neuroscience (SfN) October 17-21; Chicago, IL 2009.

39. King KE, lyemere VP, Weissberg PL, Shanahan CM: Kruppel-like factor 4 (KLF4/GKLF) is a target of bone morphogenetic proteins and transforming growth factor beta 1 in the regulation of vascular smooth muscle cell phenotype. J Biol Chem 2003, 278:11661-11669.

40. Hefferan TE, Subramaniam M, Khosla S, Riggs BL, Spelsberg TC: Cytokinespecific induction of the TGF-beta inducible early gene (TIEG): regulation by specific members of the TGF-beta family. J Cell Biochem 2000, 78:380-390.

41. Kang Y, Chen CR, Massague J: A self-enabling TGFbeta response coupled to stress signaling: Smad engages stress response factor ATF3 for Id1 repression in epithelial cells. Mol Cell 2003, 11:915-926.

42. Bouret S, De Seranno S, Beauvillain JC, Prevot V: Transforming growth factor beta1 may directly influence gonadotropin-releasing hormone gene expression in the rat hypothalamus. Endocrinology 2004, 145:1794-1801.

43. Rampon $\mathrm{C}$, Jiang $\mathrm{CH}$, Dong $\mathrm{H}$, Tang YP, Lockhart DJ, Schultz PG, Tsien JZ, $\mathrm{Hu} Y$ : Effects of environmental enrichment on gene expression in the brain. Proc Natl Acad Sci USA 2000, 97:12880-12884.

44. Segerson TP, Hoefler H, Childers H, Wolfe HJ, Wu P, Jackson IM, Lechan RM: Localization of thyrotropin releasing hormone prohormone messenger ribonucleic acid in rat brain in situ hybridization. Endocrinology 1987, 121:98-107.

45. Evans PM, Zhang W, Chen X, Yang J, Bhakat KK, Liu C: Kruppel-like factor 4 is acetylated by $\mathrm{p} 300$ and regulates gene transcription via modulation of histone acetylation. J Biol Chem 2007, 282:33994-34002.

46. Gary KA, Sevarino KA, Yarbrough GG, Prange AJ Jr, Winokur A: The thyrotropin releasing hormone (TRH) hypothesis of homeostatic regulation: implications for TRH-based therapeutics. J Pharmacol Exp Ther 2003, 305:410-416.

47. Boldyrev A, Song R, Lawrence D, Carpenter DO: Carnosine protects against excitotoxic cell death independently of effects on reactive oxygen species. Neuroscience 1999, 94:571-577. 
48. Chen YW, Zhao P, Borup R, Hoffman EP: Expression profiling in the muscular dystrophies: identification of novel aspects of molecular pathophysiology. J Cell Biol 2000, 151:1321-1336.

49. Rozen S, Skaletsky H: Primer3 on the WWW for general users and for biologist programmers. Methods Mol Biol 2000, 132:365-386.

50. Sotelo-Silveira JR, Calliari A, Kun A, Benech JC, Sanguinetti C, Chalar C, Sotelo JR: Neurofilament mRNAs are present and translated in the normal and severed sciatic nerve. J Neurosci Res 2000, 62:65-74.

51. Chalfie M, Tu Y, Euskirchen G, Ward WW, Prasher DC: Green fluorescent protein as a marker for gene expression. Science 1994, 263:802-805.

52. Sadot E, Marx R, Barg J, Behar L, Ginzburg I: Complete sequence of 3'untranslated region of Tau from rat central nervous system. Implications for mRNA heterogeneity. J Mol Biol 1994, 241:325-331.

53. Hoshimaru M, Ray J, Sah DW, Gage FH: Differentiation of the immortalized adult neuronal progenitor cell line $\mathrm{HC} 2 \mathrm{~S} 2$ into neurons by regulatable suppression of the v-myc oncogene. Proc Natl Acad Sci USA 1996, 93:1518-1523.

54. Dudoit S, Fridlyand J: A prediction-based resampling method for estimating the number of clusters in a dataset. Genome Biol 2002, 3 : RESEARCH0036.

doi:10.1186/1471-2164-12-222

Cite this article as: Guerra-Crespo et al:: Transcriptional profiling of fetal

hypothalamic TRH neurons. BMC Genomics 2011 12:222.

\section{Submit your next manuscript to BioMed Central} and take full advantage of:

- Convenient online submission

- Thorough peer review

- No space constraints or color figure charges

- Immediate publication on acceptance

- Inclusion in PubMed, CAS, Scopus and Google Scholar

- Research which is freely available for redistribution

Submit your manuscript at www.biomedcentral.com/submit
C Biomed Central 